\title{
Influence of the microresonator nonlinearity on the self-injection locking effect
}

\author{
Nikita Kondratiev ${ }^{1, *}$, Alexander Gorodnitskiy ${ }^{1,2}$, and Valery Lobanov ${ }^{1}$ \\ ${ }^{1}$ Russian Quantum Center, 121205 Moscow, Russia \\ ${ }^{2}$ Moscow Institute of Physics and Technology, 141700 Dolgoprudny, Moscow region, Russia
}

\begin{abstract}
The effect of self-injection locking of a laser to a nonlinear microresonator is considered. It is shown that an additional detuning arises in the system, depending on the pump power. This effect can contribute to the generation of optical combs in the pull mode, which will increase the stability of the generated signal.
\end{abstract}

Frequency combs are of great importance for many modern applications in science and technology. Even more interest was attracted to this field, when they were shown to exist in whispering gallery mode (WGM) microresonators [1]. One of the most important objects associated with microresonator-based frequency combs is dissipative Kerr soliton (DKS) [2] representing coherent optical frequency combs with smooth spectral profile. The DKS may only exist if the pump is red detuned from a WGM resonance to compensate Kerr frequency shift. Usually, this can be achieved by tuning the pump laser frequency from the blue to the red slope of the resonance curve.

Previous works [2] show that soliton generation happens in a certain range of normalized detunings with lower boundary being the bistability criterion and upper boundary being the soliton stability criterion $\zeta \in\left[-\frac{\pi^{2} f^{2}}{8} ;-3\left(\frac{f}{2}\right)^{\frac{2}{3}}+\frac{1}{4}\left(\frac{f}{2}\right)^{-\frac{2}{3}}\right]$ where $f$ is normalized pump, $\zeta=2\left(\omega-\omega_{\mu}\right) / \kappa$ is the detuning of the generation frequency from the microresonator eigenfrequency, normalized to the mode half-linewidth $\kappa / 2$ (half loaded decay rate). The obstacle lies in the temperature drop, the resonator experiences when the pump laser transits from the blue detuned (high intracavity power) to the red detuned (lower intracavity power) state. This sudden temperature drop leads to a blue-shift of the resonance frequency and a loss of the soliton state. On the other hand, when the tuning into the soliton state is too quick, the resonator is still cold, and its subsequent heating will again lead to a loss of the soliton state. This problem was initially solved by tuning into the soliton state with an ideal, intermediate speed of frequency scan, such that the resonator reaches the soliton state in a thermal equilibrium, that is, neither too hot nor too cold.

A simpler and more efficient method proposed in [3] and implemented in [4] is to selfinjection lock a pump laser onto a high-Q microcavity. The drag effect occurs due to Rayleigh scattering inside the microcavity and the feedback of the wave, which returns to the laser, gets a resonant coupling. It was shown in [3] that the frequency of a self-injection

\footnotetext{
*Corresponding author: noxobar@mail.ru
} 
locked laser will follow the frequency of the resonator caused by thermal effects. However, in terms of this model it can be shown that the detunings provided by the self-injection locking is too small to provide soliton generation that contradicts to experimental observations reported in [4]. To solve this problem, we noted that the comb generation assumes the nonlinearity to be essential in the microresonator and we modified selfinjection locking theory taking microresonator nonlinearity into account.

Combining the dynamics equations for WGM from [3] with nonlinear terms from [2] with arbitrary pump in stationary case we get

$$
\begin{aligned}
& f=(-1+i \zeta) a^{+}+i \beta a^{-}+i a^{+}\left(\left|a^{+}\right|^{2}+2 \alpha_{x}\left|a^{-}\right|^{2}\right) \\
& 0=(-1+i \zeta) a^{-}+i \beta a^{+}+i a^{-}\left(\left|a^{-}\right|^{2}+2 \alpha_{x}\left|a^{+}\right|^{2}\right)
\end{aligned}
$$

Here we introduced the coefficient $\alpha_{x}$, which is obtained from the mode overlap integrals and is close to 1 for modes with the same polarization and close to $1 / 3$ for different polarizations. It can be shown that after the inclusion of thermal equation and renormalizing the amplitude, we can get the same form of the equation with $\alpha_{x} \rightarrow \frac{2 \alpha_{x}+\alpha_{\theta}}{2+2 \alpha_{\theta}}$, where $\alpha_{\theta}$ is a combination of thermal relaxation times of the system. Then we showed that introduction of nonlinear detuning and nonlinear coupling, so that $\zeta \rightarrow \bar{\zeta}=\zeta+\delta \zeta_{\mathrm{nl}}$, $\beta^{2} \rightarrow \overline{\beta^{2}}=\beta^{2}+\delta \beta^{2}$ l, provides the same equations for the description of the self-injection locking effect in nonlinear microresonator as in [3] and all derivations persist for these new variables.
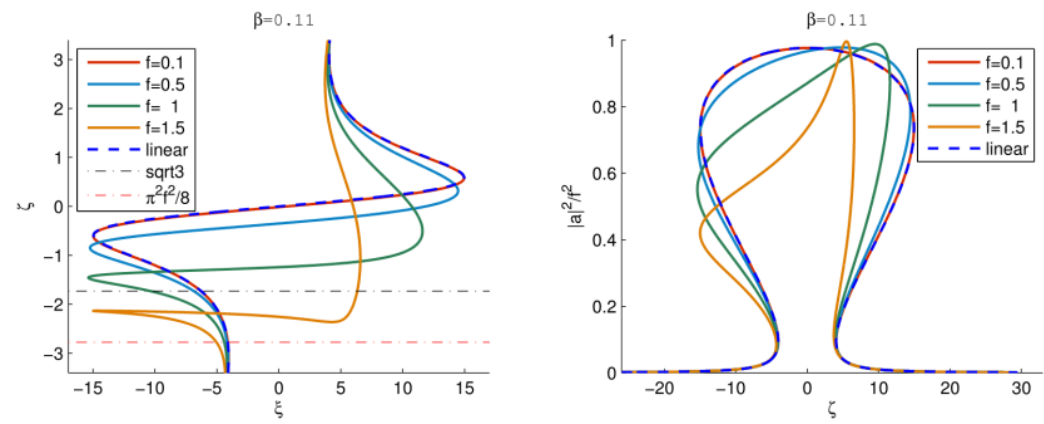

Fig. 1. Tuning (left) and resonance (right) non-linear curves of self-injection locking for different pump amplitudes (continuous lines) along with a curve for linear self-injection locking (dashed line). The boundaries of the soliton generation region (dashed-dotted line) are also plotted in the right figure.

Derived equations were solved numerically to get insights on tuning curves in nonlinear regime (fig. 1). It was confirmed that the nonlinearity provides the detuning, necessary for the soliton generation. We confirmed our predictions that the locking should help to overcome the thermal effects and showed that nonlinear frequency shift helps to reach the desired detuning and reach the soliton comb state inside the locking band.

The work was supported with RSF (project 19-72-00173)

\section{References}

1. P. Del'Haye, A. Schliesser, O. Arcizet et.al., Nature 450, 7 (2004)

2. T. Herr, V. Brasch, J. D. Jost et. al., Nat. Photon., 414, 145, (2014)

3. N. M. Kondratiev, V. E. Lobanov, A. V. Cherenkov et. al., Opt. Express, 25, 28167, (2017)

4. N. G. Pavlov, S. Koptyaev, G. V. Lihachev et.al., Nat. Photon., 12, 694 (2018) 